\title{
Complementarity in Psychophysics
}

\author{
Pierre Uzan \\ Chercheur associé au laboratoire CHArt, Cognition Humaine et Artificielle \\ pierre.uzan@paris7.jussieu.fr
}

\begin{abstract}
Besides the application of the notion of complementarity to psychological and physical descriptions of the individual, this paper explores the possibility of defining complementary observables in the same phenomenal domain. Complementary emotional observables are defined from experimental data on experienced emotions reported by subjects who have been prepared in a state of induced emotion. Complementary physiological observables are defined in correspondence with conjugate, physiological quantities that can be measured.
\end{abstract}

Keywords: Complementarity, Psychophysics, Emotional observables, Induced emotion, Physiological observables, Conjugate physiological quantities.

\section{Introduction.}

The notion of complementarity has been implemented according to different ways in quantum mechanics $[1,2,3]$. In a first sense, it applies to the descriptions of a same quantum system that exclude each other but are nevertheless required together to give a complete information on it. Mathematically speaking, a "description" can be defined as an algebra of experimental propositions and two Boolean descriptions, which are governed by classical logic, can be said "complementary" if they cannot be embedded into a single Boolean description. It is the case of the wave/corpuscle descriptions of particules which are associated with exclusive phenomena in the double-slit experiment. However, the notion of complementarity is also used to indicate the non-commutativity of physical observables, which relies on the fact that the order in which are measured the corresponding physical quantities is not indifferent. It is for example the case for the position and moment observables whose degree of complementarity is evaluated by the Planck constant.

On the other hand, the recent propositions for generalizing quantum theory have shown that its key-concepts can apply beyond the strict quantum domain, and even beyond the material realm [4]. Diverse non-physical phenomena, such as bistable perception, combination of concepts in a sentence or decision making in uncertain situation have been successfully modeled within this generalized framework, by taking into account the quantum-like effects 
that are involved -like order effects or interference effects [5,6,7]. Along this line of thought, this paper will discuss the possibility of successfully applying the notion of complementarity to psychophysics.

The idea of complementarity of mental and bodily descriptions of the individual can indeed be traced back to Spinoza's double-aspect monism. According to Spinoza, Extension and Mind are (among an infinite number of attributes) two intelligible and non-interacting aspects of the neutral substance, which is God or Nature. Spinoza's parallelism of aspects has been reinterpreted by Pauli in terms of complementarity of descriptions, which then, in agreement with Bohr's project to apply the notion of complementarity beyond physics, generalizes the notion of complementary descriptions in quantum mechanics. The latter application of the notion of complementary descriptions to the mind-matter relationship has been explored in details by Primas with a special focus on the notion of time [8]: Mc Taggart's A-time and Btime are conceived of as complementary representations of time, which can then be used to parametrize both causal chains of mental and physical events. Let us also mention the suggestion of Walach and Römer [9,10] to apply the notion of complementarity of mental and bodily descriptions of the individual to single observables, defined across domains, and not (only) to algebras of propositions. The underlying idea is that the measurement of a psychological observable generally changes the state of the subject and then the possible results of the measurement of a physiological observable -and reciprocally.

We shall here focus on the possibility of defining complementary observables in the same phenomenal domain and not across domains. The reason of this choice is that such an approach to complementarity in psychophysics can be very useful for tackling the longstanding question of the nature of the psychophysical correlations on experimental basis, by appealing to Bell-type inequalities [11]. In addition, if the complementarity of mental observables has been successfully explored in the domain of cognition and decision making [7], it seems that it is not yet the case for observables relative to the subjective experience, that is, observables that evaluate the emotional content of the mental states of a subject in a life situation. Likewise, it seems that the complementarity of physiological observables, which are defined from the macroscopic physiological quantities (such as the heart rate, the blood pressure or the skin conductance), has not yet been explored.

In section 2, complementary emotional observables will be defined from experimental data on the intensity of the emotions experienced by subjects prepared in a state of induced emotion, which then defines an emotional context. 
In contrast, a theoretical method for defining complementary physiological observables from couples of conjugate physical variables will be presented in section 3 . This method will be applied to cardiovascular quantities (systolic blood pressure and stroke volume) that can be easily measured. The conclusion and the scope of this study will be briefly discussed in the final section 4 .

\section{Complementary emotional observables.}

Emotional observables measure the degree of specified emotions experienced by a subject, such as anger, sadness, happiness, or fear. The complementarity of emotional observables will be shown by computing their commutator from the relevant experimental data. This can be done if we can compute from these data the conditional probabilities that an individual be "observed" (through a questionnaire or other means) in an emotional state if she/he has been conditioned or "prepared" in some specified emotional state by a reliable method. This is actually the case for the data provided by several recent papers, like that of Prkachin et al. [12] that will be analysed hereafter.

These authors have reported data about the measurement of cardiovascular variables of subjects experiencing emotional states induced by particular stimuli constructed according to Lang's theory [13]. These stimuli are capable of triggering relatively "pure" emotions by referring to specific incidents in their lives. In the present case, the experimenter tells the subjects stories that are directly related to these specific incidents, but other methods based on the projection of films have also been shown reliable [14]. The five considered emotional contents are here "happiness" (noted as H), "sadness" (S), "anger" (A), "fear" (F) and "disgust" (D). In order to show the reliability of the method of conditioning, Prkachin and his colleagues have measured the average reported intensity (average on a population of 31 individuals, 23 females and 8 males) of the five basic emotions experienced on the six possible trials (the five trials of induced specific emotions and a trial where no specific emotion is induced). We shall here be exclusively interested in the latter part of the data. This intensity of the experienced emotions is measured on a scale of 0 (no emotion experienced) to 7 (extremely intense emotional experience). The results, which are presented in Prkachin's paper (p. 259), are reported hereafter (table I). 
Lecture Notes in Computer Science, Springer, Vol. 9535: 168-178 (2016)

Table I--Average intensity of each of five emotions experienced on six trials

\begin{tabular}{lccccc}
\hline & \multicolumn{5}{c}{ Emotional rating } \\
\cline { 2 - 6 } Target & Happiness & Anger & Fear & Sadness & Disgust \\
\hline Neu & $0.24^{\mathrm{a}}$ & 0.03 & 0.09 & 0.15 & 0.03 \\
& $(0.66)$ & $(0.17)$ & $(0.38)$ & $(0.57)$ & $(0.17)$ \\
Hap & $\mathbf{5 . 3 0}$ & 0.03 & 0.24 & $0.21^{\mathrm{a}}$ & 0.15 \\
& $(1.36)$ & $(0.17)$ & $(0.71)$ & $(0.49)$ & $(0.87)$ \\
Ang & 0.00 & $\mathbf{5 . 1 5}$ & 0.39 & $0.81^{\mathrm{a}}$ & $1.21^{\mathrm{a}}$ \\
& $(0.00)$ & $(1.15)$ & $(1.12)$ & $(1.36)$ & $(1.62)$ \\
Fea & 0.21 & $0.73^{\mathrm{a}}$ & $\mathbf{4 . 6 1}$ & $1.09^{\mathrm{a}}$ & 0.36 \\
& $(0.74)$ & $(0.31)$ & $(1.50)$ & $(1.59)$ & $(1.08)$ \\
Sad & 0.06 & $1.27^{\mathrm{a}}$ & $0.79^{\mathrm{a}}$ & $\mathbf{5 . 1 5}$ & 0.36 \\
& $(0.24)$ & $(1.81)$ & $(1.50)$ & $(1.62)$ & $(1.06)$ \\
Dis & 0.06 & $1.58^{\mathrm{a}}$ & $0.58^{\mathrm{a}}$ & $0.85^{\mathrm{a}}$ & $\mathbf{4 . 9 7}$ \\
& $(0.24)$ & $(1.94)$ & $(0.97)$ & $(1.46)$ & $(1.40)$ \\
\hline
\end{tabular}

(from Prkachin et al. 1999)

For example, we can read on the fourth line (target emotion $=$ fear), that a subject submitted to a fear-induced stimulus will, on average, report an intensity of fear of 4.61 (with a standard deviation SD of 1.50) and an intensity of happiness of 0.21 (with $\mathrm{SD}=0.31$ ). All the data of this table show that among the reported emotions, the target emotion, induced by the trial, obtains a much higher rating than all other emotions ${ }^{1}$, which shows the reliability of the method. Nevertheless, note that the target emotion is never reported with its maximal intensity (that is, with a rating of 7), which shows that the emotional state of a subject is not totally determined by the conditioning. We will then write a state of induced emotion $B$ as $\mathrm{W}_{\mathrm{B}}$ (and not as B) to distinguish this state from the state of "pure" emotion B (which would give rise to a rating of 7).

Now, for each reported emotion, these data do not provide the number of subjects that report each of the 8 possible ratings, from 0 to 7 , but an only number, its average intensity on

\footnotetext{
1 Note that the authors interpret the latter result ontologically, by saying that the subjects are in a "mixed" emotional state even if the target emotion is nevertheless predominant. However, it seems that from a purely phenomenological approach it cannot be rigorously asserted on the basis of reports that the subject was in the same emotional states before questioning her/him. This point of view is strengthened by some recent results that show that the fact of reporting one's emotion changes the values of one's physiological variables and then of the correlated emotional variables [14].
} 
the population. We cannot then work, as is usually the case for the quantum models of cognition and decision making [7], in an 8-dimensional state space corresponding to the 8 possible weightings of each reported emotion. Nevertheless, the average intensity of each reported emotion can be used to estimate the conditional probability to report this emotion for a subject prepared in a given state of induced emotion. From table 1, the conditional probability to report the emotion A for a subject "prepared" in a state of induced emotion $\mathrm{W}_{\mathrm{B}}$ will be defined as the rate of the reported average intensity of A with respect to the sum of all the reported average intensities of emotions for subjects prepared in $\mathrm{W}_{\mathrm{B}}$. For example, the probability to report an emotion of disgust for a subject prepared in an emotional state of anger can be computed from the third line of table $\mathrm{I}$ as $\mathrm{p}\left(\mathrm{D} / \mathrm{W}_{\mathrm{A}}\right)=1.21 / 7.56=0.160$.

In order to interpret geometrically these conditional probabilities, the emotional states and the emotional observables will be represented within a 2-dimensional state space where each emotion $E$ defines the orthonormal E-basis $\{|E>|\rceil E>$,$\} , the vector \mid E>$ (written in Dirac notation) representing a state of extreme emotion $\mathrm{E}$ (with a rating of 7) and the vector $\mid 7 \mathrm{E}>\mathrm{a}$ state where no emotion $\mathrm{E}$ is felt (rating 0). This 2-dimensional state space is a complex Hilbert space capable of representing incompatible emotions (see hereafter). In this state space, an emotional state is represented by a unit vector, while emotional observables will be defined as projectors. For example, the Fear observable and the Sadness observable are respectively defined by the projectors $\mathbf{F}_{\mathrm{op}}=|\mathrm{F}\rangle\langle\mathrm{F}|$ and $\mathbf{S}_{\mathrm{op}}=|\mathrm{S}\rangle\langle\mathrm{S}|$. As shown in the following diagram, the probability amplitude that a subject reports the emotion $\mathrm{S}$ if he/she has been conditioned in a state $\mathrm{W}_{\mathrm{F}}$ of induced fear, which can be computed from the data by taking the square root of the corresponding conditional probability, will be interpreted as the magnitude of the projection of $\left|\mathrm{W}_{\mathrm{F}}\right\rangle$ onto $|\mathrm{S}\rangle$ :

$$
<\mathrm{S}\left|\mathrm{W}_{\mathrm{F}}\right\rangle_{\mathrm{df}}\left(\mathrm{P}\left(\mathrm{S} / \mathrm{W}_{\mathrm{F}}\right)\right)^{1 / 2} \equiv \text { length } \mathrm{OH} .
$$

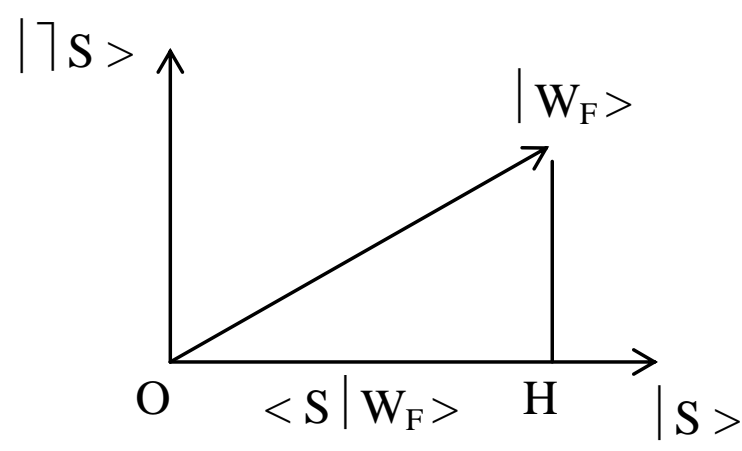


To show the complementarity of two emotional observables, we have first to represent their associated basis in the same 2-dimensional Hilbert space, then to define the corresponding emotional observables and compute their commutator. For example, let us consider the emotions Anger (A) and Disgust (D), whose associated observables are, respectively, the projectors $\mathbf{A}_{\mathrm{op}}=\mid \mathrm{A}>\langle\mathrm{A}|$ and $\mathbf{D}_{\mathrm{op}}=|\mathrm{D}\rangle\langle\mathrm{D}|$.

The following diagram shows in the 2-dimensional emotional state space the basis $\{|\mathrm{A}\rangle$, $\| \mathrm{A}>\}$ and $\{|\mathrm{D}>| \mid, \mathrm{D}>\}$ respectively associated to these emotions and the angles between these vectors and the state of induced anger $\left|\mathrm{W}_{\mathrm{A}}\right\rangle$ that will be used to compute the components of the vector $\mid \mathrm{D}>$ in the basis $\{|\mathrm{A}>| \mid, \mathrm{A}>\}$ :

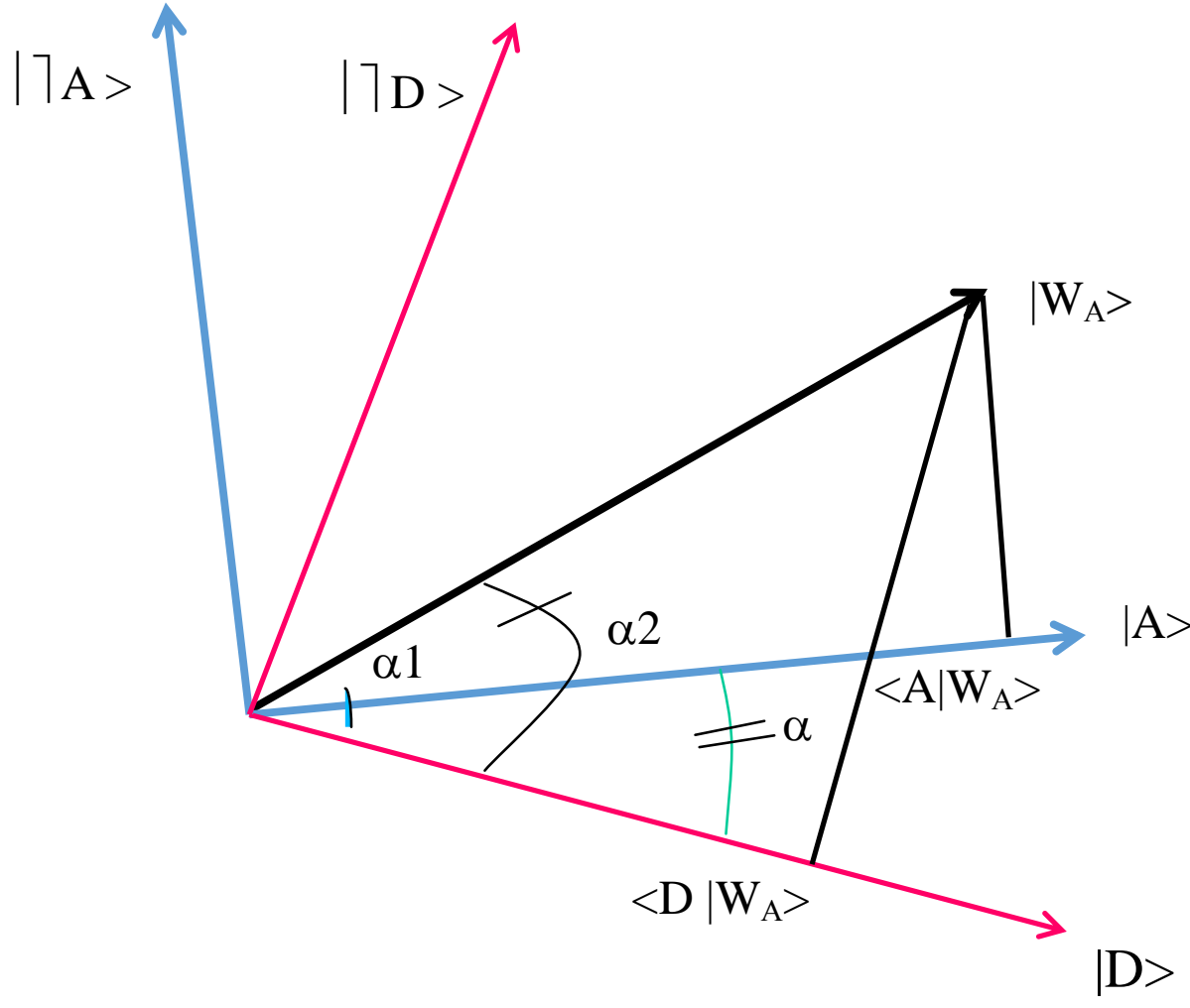

The probability amplitude $\langle\mathrm{D} \mid \mathrm{A}\rangle$ of the transition from the state of pure anger to the state of pure disgust can be computed as follows:

$\cos \alpha 1=\left\langle\mathrm{A} \mid \mathrm{W}_{\mathrm{A}}\right\rangle=(5.15 / 7.56)^{1 / 2}=0.825$ (see line 3$)$, hence $\alpha 1=38.2^{\circ}$

$\cos \alpha 2=\left\langle\mathrm{D} \mid \mathrm{W}_{\mathrm{A}}\right\rangle=\left(\mathrm{p}\left(\mathrm{D} / \mathrm{W}_{\mathrm{A}}\right)\right)^{1 / 2}=0.4$ (see above), hence $\alpha 2=73,8^{\circ}$

$\alpha=\alpha 2-\alpha 1=35.6^{\circ}$, and then $\langle\mathrm{A} \mid \mathrm{D}\rangle=\cos \alpha=0.847$.

Now, the state $\mid \mathrm{D}>$ can be written in the A-basis as:

$$
|\mathrm{D}\rangle=\langle\mathrm{A} \mid \mathrm{D}\rangle|\mathrm{A}\rangle+\langle\rceil \mathrm{A}|\mathrm{D}\rangle|| \mathrm{A}\rangle,
$$

where the probability amplitude \langle\rceil $\mathrm{A}|\mathrm{D}\rangle$ can be computed from $\langle\mathrm{A} \mid \mathrm{D}\rangle$ as follows:

$$
\left.<\rceil \mathrm{A}|\mathrm{D}\rangle=\left.(1-|<\mathrm{A}| \mathrm{D}\rangle\right|^{2}\right)^{1 / 2}=0.531 \text {. }
$$


Consequently, $|\mathrm{D}\rangle=0.847|\mathrm{~A}\rangle+0.531|| \mathrm{A}\rangle$.

To compute the commutator of the observables $A_{o p}$ and $D_{o p}$, we will express their associated matrix in the same orthonormal basis $\{|\mathrm{A}\rangle, \mid\rceil \mathrm{A}\rangle\}$ of the two-dimensional state space. In this basis, the projector $\mathrm{A}_{\mathrm{op}}=|\mathrm{A}\rangle\langle\mathrm{A}|$ is associated with the matrix:

$$
\begin{aligned}
& M\left(A_{\text {op }}\right)=\left\lceil\begin{array}{ll}
1 & 0
\end{array}\right\rceil \\
& \left\lfloor\begin{array}{ll}
0 & 0
\end{array}\right\rfloor
\end{aligned}
$$

To find the matrix associated with the observable $\mathbf{D}_{\text {op }}$ in the basis $\left.\left.\{|A>|\rceil A,\right\rangle\right\}$ we use the aforementioned decomposition of $|\mathrm{D}\rangle$ in the basis $\{|\mathrm{A}\rangle, \mid\rceil \mathrm{A}\rangle\}$ to compute the outer product $\mathbf{D}_{\mathrm{op}}=|\mathrm{D}\rangle\langle\mathrm{D}|$ in this basis:

$$
\begin{aligned}
& \mathrm{M}\left(\mathbf{D}_{\text {op }}\right)=(0.847 ; 0.531) \otimes(0.847 ; 0.531)=\lceil 0.717 \quad 0.449\rceil \\
& \left\lfloor\begin{array}{ll}
0.449 & 0.282
\end{array}\right.
\end{aligned}
$$

The commutator of the observables $\mathbf{A}_{\mathrm{op}}, \mathbf{D}_{\mathrm{op}}$ can then be computed as:

$$
\begin{aligned}
& {\left[\mathbf{A}_{\text {op }}, \mathbf{D}_{\text {op }}\right]=\left\lceil\begin{array} { l l } 
{ 1 } & { 0 }
\end{array} \left\lceil\begin{array}{ll}
0.717 & 0.449
\end{array}-\lceil 0.717 \quad 0.449\rceil\lceil 1 \quad 0\rceil\right.\right.}
\end{aligned}
$$

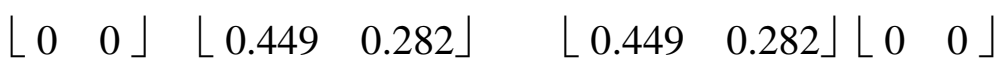

$$
\begin{aligned}
& =(0.449)\lceil 0 \quad 1\rceil . \\
& \lfloor-1 \quad 0\rfloor
\end{aligned}
$$

This result shows the complementarity of the observables $\boldsymbol{A}_{\text {op }}$ and $\boldsymbol{D}_{\text {op }}$ that, respectively, measure the average intensity of anger and disgust experienced by a subject conditioned in a state of induced anger. Their degree of incompatibility can be estimated by the norm of this commutator:

$$
\text { | }\left[\mathbf{A}_{\mathrm{op}}, \mathbf{D}_{\mathrm{op}}\right]||=0.449 \text {. }
$$

It can be shown that all couples of emotions involved in these data are complementary. Moreover, many other examples of complementary emotional observables can be provided from the relevant experimental data reported in the literature (for example, from the papers by Kassam and Mendes [14], Pauls and Stemmler [15] and by Sinha et al. [16]).

\section{Complementary physiological observables.}

In the physiological domain, it seems that no data about successive measurements of quantities or measurements of quantities in different specified contexts (as is the case in section 2) has been systematically reported. We will then use a theoretical method to define 
couples of complementary observables that generalizes the one which is used for defining conjugate observables in standard quantum theory (like position and momentum). The underlying idea is that conjugate variables of classical physics (like pressure and volume, pulsation and time,...) can be used to define complementary physiological observables because, as it can be shown, their measurements are interdependent. This method can be applied to several couples of cardiovascular observables that are involved in the relevant data.

Let us first give an example of conjugate variables that will be used hereafter to define complementary physiological observables. To say that the variables $\mathrm{p}$ (pressure) and $\mathrm{v}$ (volume) are conjugate variables in the thermodynamic description of any fluid means that they are always associated to each other, as a product of a generalized "force" (intensive variable) and a generalized "displacement" (extensive variable), in the calculation of the change of the internal energy of this system - as is also the case for the couples temperature/entropy and chemical potential/ number of molecules:

$$
\mathrm{d} U=-\mathrm{pdv}+\mathrm{T} \mathrm{ds}+\Sigma_{\mathrm{i}} \mu_{\mathrm{i}} \mathrm{dn}_{\mathrm{i}}+\ldots
$$

Now, the couple of conjugate variables $\mathrm{p}, \mathrm{v}$ can be used for defining conjugate cardiovascular observables in as much as they are involved in the definitions of the blood pressure and the stroke volume, which is the volume of blood pumped the heart in a cycle. These cardiovascular variables can be easily measured as shown by the relevant physiological studies and, particularly for our purpose, in many psychophysical studies (for example, see $[12,14,15,16])$. The interdependency of the measurements of the blood pressure and the stroke volume in a cardiac cycle can be described as follows: To measure the volume of blood in a ventricle of the heart, we send ultrasounds on it (echography), which exert a pressure on this cavity and then change the blood pressure. Reciprocally, to measure the blood pressure, we change the blood flow by exerting a pressure on the arm's artery (by using a cuff filled with air and progressively emptied), which changes the volume of blood pumped by the heart.

This idea of interdependency of the measurement of physiological quantities can be implemented by defining complementary physiological observables acting on the physiological state space. Due to the fact that physiological quantities are continuous, this state space is here an infinite-dimensional complex Hilbert space $\mathrm{H}$ spanned by the vectors associated to the possible values (which are supposed non-degenerated) of all physiological quantities. The physiological state of a subject is represented by a unit vector of the state space $\mathrm{H}$. 
To implement the previous example, let us first define a pressure observable, which computes the blood pressure of the left ventricle of the heart of a subject in state $|W\rangle$ of $H$. This observable can be defined as:

$$
\left\langle\mathrm{p}\left|\mathbf{P}_{\text {op }}\right| \mathrm{W}\right\rangle=_{\text {df }} \mathrm{p}\langle\mathrm{p} \mid \mathrm{W}\rangle
$$

in the "p-representation", that is, in the continuous basis $\{\mid \mathrm{p}>\}$ of the eigenvectors of $\mathbf{P}_{\mathrm{op}}$ associated with its possible values. A straightforward correspondence can be established between the expectation value of the pressure observable $\mathbf{P}_{\mathrm{op}}$ and the systolic blood pressure (SBP), which is the maximal blood pressure that can be measured:

$$
\mathrm{SBP}=\left\langle\mathbf{P}_{\mathrm{op}}\right\rangle_{\mathrm{ED}}
$$

where ED, the end of diastole state, specifies the moment of the cardiac cycle when the blood pressure is at its maximum value.

Let us now consider a volume observable, defined as:

$$
<\mathrm{v}\left|\Delta \mathbf{V}_{\text {op }}\right| \mathrm{W}>=_{\text {df }}\left(\mathrm{v}-\mathrm{v}_{\mathrm{ES}}\right)<\mathrm{v} \mid \mathrm{W}>,
$$

where $\mathrm{v}_{\mathrm{ES}}$ is the end-systolic volume of the left ventricle of the heart (which is the minimal volume of blood in this ventricle). This observable measures the increasing in the volume of blood contained in this ventricle of the heart with respect to its minimal value $\mathrm{v}_{\mathrm{ES}}$. The interest of this definition is that the stroke volume $(\mathrm{SV})$, which is the difference $\left(\mathrm{v}_{\mathrm{ED}}-\mathrm{v}_{\mathrm{ES}}\right)$, is equal to the expectation value $\left\langle\Delta \mathbf{V}_{\text {op }}\right\rangle$ of the observable $\Delta \mathbf{V}_{\text {op }}$ at the same end of diastole state:

$$
\mathrm{SV}=\left\langle\Delta \mathbf{V}_{\mathrm{op}}\right\rangle_{\mathrm{ED}}
$$

To show the complementarity of $\mathbf{P}_{\mathrm{op}}$ and $\Delta \mathbf{V}_{\text {op }}$, the pressure and volume observables, we have first to show that the p-representation $\langle\mathrm{p} \mid \mathrm{W}\rangle$ and the v-representation $\langle\mathrm{v} \mid \mathrm{W}\rangle$ of any physiological state $|\mathrm{W}\rangle$ are Fourier transforms. This can be done by noting that $\langle\mathrm{p} \mid \mathrm{W}\rangle$ and $<\mathrm{v}|\mathrm{W}\rangle$ are square-summable functions that admit (by Plancherel theorem) a Fourier decomposition. The phase of the harmonic functions evolved in these Fourier decompositions can be estimated, for a couple of blood pressure $\mathrm{p}$ and an additional volume $\Delta \mathrm{v}$ of the left ventricle of the heart, as the rate of $\mathrm{p} . \Delta \mathrm{v}$ to its maximal value $\Delta \mathrm{U}$. The latter quantity $\Delta \mathrm{U}$ is the mechanical work of the heart in a cycle when the temperature remains constant and no chemical reactions take place (which is indeed the case here). Due to homeostasis, $\Delta U$ can be considered as a constant for a given subject [17] and, taking into account that the right ventricle pressure is about seven time smaller than the systolic pressure SBP, it can be calculated as [18]:

$$
\Delta \mathrm{U}=1.14 \mathrm{SBP} \times \mathrm{SV} .
$$

Consequently, one can write $\langle\mathrm{v}| \mathrm{W}>$ and $\langle\mathrm{p}| \mathrm{W}>$ as Fourier transforms: 


$$
\begin{aligned}
& <\mathrm{v}\left|\mathrm{W}>=(\varepsilon)^{-1 / 2} \int_{\mathrm{p}}<\mathrm{p}\right| \mathrm{W}>\exp 2 \pi \mathrm{i} / \varepsilon(\mathrm{p} \cdot \Delta \mathrm{v}) \mathrm{d} \mathrm{p} \\
& <\mathrm{p} \mid \mathrm{W}>=(\varepsilon)^{-1 / 2} \int_{\mathrm{v}}\langle\mathrm{v}| \mathrm{W}>\exp -2 \pi \mathrm{i} / \varepsilon(\mathrm{p} . \Delta \mathrm{v}) \mathrm{dv}
\end{aligned}
$$

where $\varepsilon=\Delta \mathrm{U} / 1.14$.

Taking now into account, from the expression of $\langle\mathrm{v} \mid \mathrm{W}\rangle$ above, that $\langle\mathrm{v} \mid \mathrm{p}\rangle$ can be computed as:

$$
\langle\mathrm{v} \mid \mathrm{p}\rangle=(\varepsilon)^{-1 / 2} \exp 2 \pi \mathrm{i} / \varepsilon(\mathrm{p} . \Delta \mathrm{v})
$$

the action of the observable $\mathrm{P}_{\mathrm{op}}$ in the v-representation can be computed as:

$$
\begin{aligned}
\left\langle\mathrm{v}\left|\mathbf{P}_{\mathrm{op}}\right| \mathrm{W}\right\rangle & =\int_{\mathrm{p}}\langle\mathrm{v} \mid \mathrm{p}\rangle\left\langle\mathrm{p}\left|\mathbf{P}_{\mathrm{op}}\right| \mathrm{W}\right\rangle \mathrm{dp} \\
& =(\varepsilon)^{-1 / 2} \int_{\mathrm{p}} \mathrm{p}\langle\mathrm{p} \mid \mathrm{W}\rangle \exp 2 \pi \mathrm{i} / \varepsilon(\mathrm{p} . \Delta \mathrm{v}) \mathrm{dp} .
\end{aligned}
$$

In the right member of this equation one can recognize (to within a constant factor) the Fourier transform of $\mathrm{p}\langle\mathrm{p} \mid \mathrm{W}\rangle$, which is the derivative of $\langle\mathrm{v} \mid \mathrm{W}\rangle$ regarding the variable $\mathrm{v}$. Consequently:

$$
\left\langle\mathrm{v}\left|\mathbf{P}_{\text {op }}\right| \mathrm{W}\right\rangle=-\mathrm{i} \varepsilon \partial / \partial \mathrm{v}\langle\mathrm{v} \mid \mathrm{W}\rangle,
$$

and then $\Delta \mathrm{V}_{\mathrm{op}}$ and $\mathrm{P}_{\mathrm{op}}$ are conjugate observables:

$$
\left[\Delta \mathbf{V}_{\text {op }}, \mathbf{P}_{\text {op }}\right]=\text { i } \varepsilon \mathbf{I d},
$$

their degree of incompatibility $\varepsilon$ characterizing the cardiovascular cycle of the subject under consideration.

This method can of course be applied to other couples of conjugate physical variables that refer to complementary descriptions of a same physiological phenomenon, as is the case for the temporal and the spectral descriptions of the heart beat.

\section{Conclusion and prospects.}

This meta-analysis shows that the notion of complementarity can be implemented in psychophysics, regarding the subjective experience and regarding the physical description of the individual. Emotional, complementary observables have been defined by referring to experimental data on measurements of experienced emotions reported by subjects prepared in a given state of induced emotion. In the physiological domain, complementary observables corresponding to cardiovascular quantities can be defined by generalising the notion of conjugate observables in quantum mechanics. As has been mentioned, the few examples presented in this paper can be easily completed by considering several couples of psychological and physiological observables defined from other independent data, for 
example from those provided by Kassam and Mendes [14], Kriebig et al. [19], Paul and Stemmler [15] or Sinha et al. [16].

Beyond this conclusion, the possibility of defining couples of complementary psychological observables and complementary physiological observables paves the way for moving forward the difficult question of the nature of psychophysical correlations on experimental grounds, when the metaphysically-based strategies seem to lead to a dead end (see, for example, the analysis of Kim [20] or Esfeld [21]). This experimental test appeals to a statistical approach based on inequalities that generalize both Bell and Tsirelson inequalities. As explained by Uzan [11] from an idea originally suggested by Primas and Atmanspacher [22], the nature of the psychophysical correlations can be decided by comparing the CHSH correlation factor between couples of complementary psychological observables and complementary bodily observables with a signalling bound (which is a function of the commutators of the emotional and physiological observables) and with the Bell bound. This work can be achieved by referring to data such as those provided in the previously quoted articles, which report the change scores of cardiovascular variables of subjects experiencing emotional states. In particular, this approach could justify on experimental grounds that the psychophysical correlations are to be conceived of as relations of generalized entanglement. The work presented in this paper is then a first step for tackling the mind-body problem according to an approach which has never been applied in the field of psychophysics and can prove very fruitful.

\section{References}

[1] N. Bohr (1933-1958). Complementarity: Bedrock of the Quantal Description, in Foundations of Quantum Physics II (1933-1958), Collected Works 7, Ed. J. Kalckar, Elsevier, pp. 284-285, 1996.

[2] P. Bernays. Uber die Ausdehnung des Begriffes der Komplementarit"at auf " die Philosophie. Synthese VII, 66-70, 1948.

[3] H. Atmanspacher. Dual-aspect monism à la Pauli and Jung, Journal of Consciousness Studies Vol. 19 (9-10): 96-120, 2012.

[4] H. Atmanspacher, H. Römer and H. Walach. Weak quantum theory: Complementarity and entanglement in physics and beyond. Foundations of Physics 32: 379-406, 2002.

[5] H. Atmanspacher and T. Filk. The Necker-Zeno Model for Bistable Perception. Topics in Cognitive Science 5(4): 800-817, 2013. 
[6] D. Aerts, L. Gabora, S. Sozzo and T. Veloz. Quantum structure in cognition: Fundamentals and Applications. ArXiv: 1104.3344v1 [cs.AI], 2011.

[7] J.R. Busemeyer and P. Bruza. Quantum Models of Cognition and Decision. Cambridge University Press, 2012.

[8] H. Primas. Time-entanglement between mind and matter. Mind and Matter 1: 81-119, 2003.

[9] H. Walach. The complementarity model of brain-body relationship. Medical Hypothesis 65: 380-388, 2005.

[10] H. Walach and H. Römer. Complementarity of Phenomenal and Physiological Observables: A Primer on Generalised Quantum Theory and Its Scope for Neuroscience and Consciousness Studies. In Neuroscience, Consciousness and Spirituality. Springer. pp 97-107, 2011.

[11] P. Uzan. On the Nature of Psychophysical Correlations. Mind and Matter 12(1): 7-36, 2014.

[12] K.M. Prkachin, R.M. Williams-Avery, C. Zwa and D.E. Mills. Cardiovascular Changes During Induced Emotion: An Application of Lang's Theory of Imagery. Journal of Psychosomatic Research, Vol. 47, No. 3: 255-267, 1999.

[13] P.J. Lang. A bio-informational theory of emotional imagery. Psychophysiology 16:495$512,1979$.

[14] K.S. Kassam and W.B. Mendes. The Effects of Measuring Emotion: Physiological Reactions to Emotional Situations Depend on wether Someone Is Asking. Plos One, June 2005 .

[15] C.A. Paul and G. Stemmler. Repressive and Defensive Coping During Fear and Anger. Emotion, Vol. 3 (3): 284-302.

[16] R. Sinha, W.R. Lovallo and O.A. Parsons. Cardiovascular Differentiation of emotions. Psychosomatic Medicine 54: 422-435, 1992.

[17] R.S. Balaban. Metabolic homeostasis of the heart. Perspectives on: SGP Symposium on Mitochondrial Physiology and Medicine, May 28, 2012.

[18] M. Uehara and S.K. Koibuchi. Thermodynamics of the Heart, in Application of Thermodynamics to Biological and Materials Science, ed. M. Tadashi, InTech 2011.

[19] S.D. Kreibig, F.H. Wilhelm, W. Roth and J.J. Gross. Cardiovascular, electrodermal, and respiratory response patterns to fear- and sadness-induced films. Psychophysiology 44: 787806, 2007.

[20] J. Kim. Physicalism, or something near enough. Princeton University Press, 2005. 
Lecture Notes in Computer Science, Springer, Vol. 9535: 168-178 (2016)

[21] M. Esfeld. La philosophie de l'esprit. Paris : Armand Colin, 2005.

[22] H. Atmanspacher. Mind and matter as asymptotically disjoint, inequivalent representations with broken time-reversal symmetry, BioSystems 68:19-30, 2003. 